Cahiers d'études italiennes

\title{
Boccace dans Boiardo
}

Patrizia De Capitani

\section{OpenEdition \\ Journals}

Édition électronique

URL : http://journals.openedition.org/cei/869

DOI : 10.4000/cei.869

ISSN : 2260-779X

Éditeur

UGA Éditions/Université Grenoble Alpes

Édition imprimée

Date de publication : 15 juillet 2008

Pagination : 63-97

ISBN : 978-2-84310-122-9

ISSN : 1770-9571

Référence électronique

Patrizia De Capitani, « Boccace dans Boiardo », Cahiers d'études italiennes [En ligne], 8| 2008, mis en ligne le 15 janvier 2010, consulté le 30 avril 2019. URL : http://journals.openedition.org/cei/869 ; DOI $10.4000 /$ cei.869 


\title{
BOCCACE DANS BOIARDO
}

\author{
Patrizia De Capitani \\ Université Stendhal Grenoble 3
}

Analyser dans un article l'influence exercée par l'œuvre de Boccace sur l'Inamoramento de Orlando de Matteo Maria Boiardo, poème de 36000 vers environ, tient de la gageure. Une telle recherche est toutefois facilitée par les études de Raffaele Donnarumma et par l'index des œuvres ayant inspiré Boiardo dans la rédaction de son chef-d'œuvre ${ }^{1}$. Cet index, qui figure à la suite de l'édition critique de l'Inamoramento par Antonia Tissoni Benvenuti et Cristina Montagnani, offre un tableau précis et riche de l'influence de la production de Boccace sur le roman chevaleresque du comte de Scandiano ${ }^{2}$. C'est à partir de ces instruments critiques et bibliographiques que nous entendons revenir sur la question de l'apport de certaines œuvres de Boccace à la constitution du poème chevaleresque de Boiardo.

Un coup d'œil rapide à l'index cité permet tout de suite de voir que parmi les œuvres de Boccace qui ont le plus marqué l'Inamoramento il y a les deux poèmes Filostrato et Teseida, ainsi que certaines nouvelles du Decameron. Des coïncidences autant d'ordre matériel que littéraire nous encouragent également à circonscrire notre étude à l'impact que Filostrato, Teseida et quelques passages du Decameron ont pu avoir sur

1. Raffaele Donnarumma, "Presenze boccacciane nell'Innamorato», Rivista di Letteratura Italiana, X, 1992, p. 513-597; IDEM, Storia dell'Orlando innamorato. Poetiche e modelli culturali in Boiardo, Lucques, Maria Pacini Fazzi Editore, 1996.

2. Matteo Maria BoIARDO, Opere, tome 1, L'Inamoramento de Orlando, éd. Antonia Tissoni Benvenuti - Cristina Montagnani, Milan-Naples, Ricciardi, 1999 (parties I et II). Les index, dont celui des œuvres, ont été établis par Anna Montanari. Nous utilisons indifféremment les termes de "poème» et de «roman" chevaleresque pour désigner l'œuvre de Boiardo. Nos citations d'après l'Inamoramento proviennent de l'édition critique citée.

3. Giulio BerTONI, La Biblioteca estense e la cultura ferrarese ai tempi del duca Ercole I (14711505), Turin, Loescher, 1903. On trouve mention de Filostrato (Appendice I «Frammento di un catalogo della Libreria di Borso d'Este», 1467), de Filocolo et du Decameron (Appendice $\mathrm{II}^{2}$ "La libreria d'Ercole I 1495»), au numéro 465 de l'Appendice II $^{2}$ est mentionné un "Teseo in vulgare». 
l'œuvre majeure du comte de Scandiano. Certaines des œuvres de Boccace figuraient dans l'inventaire de la bibliothèque des Este rédigé sous Hercule I ${ }^{\text {er }}$ (1471-1505)3. L'édition de Teseida, avec commentaire de Pier Andrea de' Bassi, parut à Ferrare en 1475, alors que Boiardo était plongé dans la rédaction de l'Inamoramento. Les deux poèmes de Boccace ont en commun avec celui du comte de Scandiano cette ottava rima que l'auteur du Decameron a largement contribué à répandre et à perfectionner, s'il n'en a pas été l'inventeur ${ }^{4}$. Dans Teseida, composé entre 1340 et 1341, sont mis côte à côte pour la première fois dans la tradition italienne un récit de sujet épique et un autre de sujet amoureux, le premier servant de toile de fond au second qui est largement dominant ${ }^{5}$. Ce type d'association, cela est bien connu, est également à la base du poème de Boiardo. Filostrato, rédigé en 1339 ou quelques années plus tôt, raconte quant à lui l'amour malheureux de Troiolo pour Criseida qui n'est pas sans rappeler celui d'Orlando pour Angelica ${ }^{6}$.

Avec l'aide des précieux instruments mentionnés plus haut, nous verrons le rôle que toutes ces trois œuvres, chacune selon sa spécificité, ont pu jouer sur les amours et les armes, les deux thématiques constitutives du poème de Boiardo, sachant qu'en ce qui concerne les armes seulement l'influence de Teseida est concernée. Ce dernier poème a également joué un rôle déterminant dans l'évolution des exordes de l'Inamoramento, aspect que l'on prendra également en considération. Nous terminerons par quelques remarques d'ordre formel concernant l'appropriation et la réutilisation par Boiardo d'éléments lexicaux et expressifs de Boccace. Il est bien évident que l'influence de l'œuvre de ce dernier sur

4. Sur la question de la paternité de l'ottava rima, voir Alberto LIMENTANI, «Struttura e storia dell'ottava rima", Lettere italiane, XIII, 1961, p. 20-77; Carlo DiONISOTTI, "Appunti su antichi testi", Italia medioevale e umanistica, VII, 1964, p. 99-131; Aurelio RONCAGLIA, "Per la storia dell'ottava rima», Cultura neolatina, XXV, 1965, p. 5-14; Michelangelo PICONE, «Boccaccio e la codificazione dell'ottava", in Boccaccio: secoli di vita, éd. M. Cottino-Jones \& E.F. Tuttle, Ravenne, Longo, 1977, p. 53-65; Guglielmo GoRNI, "Un'ipotesi sull'origine dell'ottava rima», Metrica, I, 1978, p. 79-94; I Cantari. Struttura e tradizione, ed. M. Picone \& M. Bendinelli Predelli, Florence, Olschki, 1984 (avec des contributions de D. De Robertis, A. Balduino, A. Limentani, A. Bettarini Bruni).

5. Comme dans l'Inamoramento, la femme aimée ira, aussi dans Teseida, à celui qui se sera distingué au combat, simple tournoi dans Teseida, véritable affrontement entre chrétiens et infidèles chez Boiardo.

6. Luigi Surdich fixe à 1339 la date de rédaction de Filostrato, qui suivrait ainsi de près Filocolo, terminé en 1338, voir Giovanni BoCCACCIO, Filostrato, éd. L. Surdich, Milan, Mursia, 1990, « Introduzione», p. 5 et 13. Vittore Branca propose en revanche la date de 1335, voir Filostrato, in Tutte le opere di Giovanni Boccaccio, Milan, Mondadori, 1964, vol. II, p. 5.Nos citations d'après Filostrato et Teseida proviennent de cette dernière édition. 
l'Inamoramento n'est plus à prouver, en revanche il est encore possible de travailler sur la manière dont Boiardo a repris Boccace et, surtout, sur son rôle spécifique par rapport à d'autres sources. C'est ce que nous essayerons de faire à partir d'un angle d'observation bien délimité.

\section{Les amours}

On prendra tout d'abord en considération les circonstances où l'amour se manifeste, les effets de la passion sur ceux qui en sont frappés, les moyens mis en œuvre pour obtenir l'objet du désir, la conquête amoureuse et l'érotisme.

Partons de la révélation de l'amour et observons ce qu'il en est chez Boccace et chez Boiardo. Malgré les circonstances différentes, autant les personnages des poemetti de Boccace - Troiolo dans Filostrato, Arcita et Palemone dans Teseida - que ceux de Boiardo - notamment Orlando et Prasildo, le héros de la nouvelle la plus fameuse de l'Inamoramento - sont frappés par l'amour alors qu'ils ne l'attendent pas. Ce foudroiement s'exprime dans les divers ouvrages de manière variée, mais partout il est présenté comme un choc dont la victime a du mal à se relever. Celui qui incarne le mieux cette condition de "foudroyé" par l'amour est peut-être Prasildo qui sent l'amour surgir en lui alors qu'il tient sa tête sur les genoux de Tisbina pour les nécessités d'un jeu en société:

Standoli in grembo sente sì gran foco

Nel cor, che non l'avrebe mai pensato,

Per non indovinar mette ogni cura,

Ché de levarsi quindi avìa paura. (Inamoramento, I, XII, 8, 6-8)

Il est si abasourdi par ce qui lui arrive qu'il n'a pas même la force de se relever; sa position, la tête dans le giron de la femme aimée, traduit physiquement son assujettissement spirituel à Tisbina. Une réaction analogue est éprouvée par Tito, l'un des héros d'une nouvelle du Decameron dont s'est largement inspiré Boiardo pour l'histoire de Prasildo, à la vue de Sofronia, fiancée de son meilleur ami, tout comme Tisbina est déjà liée à Iroldo: «Tito [...] la cominciò attentissimamente a riguardare; e ogni parte di lei smisuratamente piacendogli [...], senza alcun sembiante mostrarne, di lei s'accese quanto alcuno amante di donna s'accendesse già mai ${ }^{7}$ ".

7. Giovanni BocCACCiO, Decameron, X, 8, 13, éd. V. Branca, Turin, Einaudi, 1992, 2 vol. Il s'agit de notre édition de référence d'où proviennent toutes nos citations. 
Autant Troiolo qu'Orlando veulent cacher leur sentiment aux yeux du monde, ayant tous les deux la réputation d'être insensibles à l'amour, mais les analogies s'arrêtent là car il n'y a rien de commun dans les deux ouvres dans la manière de représenter ce motif. Chez Troiolo, la décision de cacher ses sentiments aux autres est le fruit d'une prudente réflexion ${ }^{8}$, alors que le narrateur de l'Inamoramento nous montre les gauches tentatives d'Orlando pour dissimuler son trouble:

Ma sopra tutti Orlando a lei [Angelica] s'accosta

Col cor tremante e con vista cangiata,

Benché la voluntà tenia nascosta. (I, I, 29, 4-6)

La honte d'Orlando devient un motif comique, une composante essentielle de la gaucherie amoureuse que Boiardo prête à son personnage. Et pour cause, car en tombant amoureux d'Angelica, Orlando dément une tradition littéraire séculaire qui en avait fait le champion chaste et exemplaire de la foi et de l'Empire. Les modèles de la description d'Orlando touché par l'amour se trouvent plutôt dans la tradition chevaleresque en vers que chez Boccace. Notamment dans l'Innamoramento di Carlo Magno, poème en ottava rima paru à Venise en 1481, mais circulant déjà avant cette date, et que le comte de Scandiano connaissait ${ }^{9}$. Dans ce poème également Orlando réagit avec honte et embarras aux avances d'une belle païenne qui, après l'avoir embrassé, s'offre à lui avec désinvolture:

«Vedi, bel cavallier, se son zoiosa,

Del mio amor farai che ti conforti,

El primo e' tu che prenderai la rosa,

Deh, non mi abandonar, non mi far torti»

Orlando con sua faza vergognosa

In altra parte avia li ochi torti,

E fortemente si aveva a vergognare,

Essere vorrebe mo de qua dal mare ${ }^{10}$.

Le médiocre auteur de l'Innamoramento di Carlo Magno ne va pas plus loin dans la transformation du personnage d'Orlando qui reste, dans cette

8. Filostrato, I, 36.

9. Marco VILlORESI, La Fabbrica dei cavalieri. Cantari, poemi, romanzi in prosa fra Medioevo e Rinascimento, Rome, Salerno Editrice, 2005, p. 138-139. Boiardo connaissait l'Innamoramento di Carlo Magno, car il cite le nom de la femme aimée par celui-ci, Belisandra, dans son poème (cf. Inamoramento, I, XXVIII, 5, 1).

10. Innamoramento di Carlo Magno, c. XL, cité par Rosanna Alhaique PetTinelli, "L'Orlando innamorato e la tradizione cavalleresca in ottave. I. Raffronti di personaggi e situazioni ", Rassegna della Letteratura Italiana, 71, 1967, p. 386. 
œuvre, le guerrier pudique de la tradition carolingienne, mais il offre incontestablement une amorce que le comte de Scandiano saisit et développe avec art. L'Innamoramento di Carlo Magno, qui connut un remarquable succès à la fin du XV $\mathrm{X}^{\mathrm{e}}$ siècle, témoigne de la vogue que rencontrait à cette époque la parodie des personnages carolingiens ${ }^{11}$. Or, on sait bien que le renversement parodique de certains sujets et caractères anticipe souvent un renouvellement plus profond et sérieux d'un genre tel celui que Boiardo promeut avec le roman chevaleresque.

Pour en rester à la tradition carolingienne, le comte de Scandiano disposait toutefois d'autres modèles plus aboutis pour renouveler la psychologie amoureuse de son héros éponyme. Nous pensons notamment à l'Entrée d'Espagne, poème franco-italien, composé par un auteur anonyme de la région de Padoue, remontant à la première moitié du XIV siècle. Ici, le héros incorruptible du courant carolingien perd la tête pour la belle Dionès, fille du sultan de Perse: «Rolant la garde, trestot le sang li mue; / Non la voudroit le ber avoir veüe; / D'Audein li mambre, tot le vis li tresve $^{12} »$. Ce n'est qu'un instant, car il se ressaisit tout de suite aux dépens de la belle, consciente de n'avoir aucune chance, car «Rolant s'estoit mis in autre convoitisse ${ }^{13}$ ", mais c'est sérieux. Car le Rolant de l'Entrée n'est pas un pantin sans nuances comme celui de l'Innamoramento di Carlo Magno, mais un héros profondément croyant qui vit dans la douleur son dilemme, quoique fugace, entre son devoir et l'amour ${ }^{14}$. Nous savons par ailleurs que sans le poème de l'anonyme padouan n'existeraient ni le poème de Pulci, ni ceux de Boiardo et de l'Arioste. L'exemple de l'Entrée nous rappelle que le renouvellement du roman chevaleresque entrepris par Boiardo se fonde sur la rencontre entre les formes populaires de la tradition en ottava rima et des ouvres appartenant à la haute littérature dont celles de Boccace et de l'anonyme padouan.

Un autre élément important à considérer est l'apparition de la femme aimée. Dans Filostrato, Criseida apparaît à Troiolo, comme nous l'avons anticipé, lors d'une cérémonie religieuse et il est touché, plus que par sa

11. VILlORESI, La Fabbrica..., p. 148-154.

12. L'Entrée d'Espagne. Chanson de geste franco-italienne publiée d'après le manuscrit unique de Venise, par Antoine Thomas, Paris, Firmin Didot, 1913, 2 vol., DXLII, v. 12562-12564.

13. Ibid., DLXXX, v. 13675.

14. Voici comment Dionès apparaît aux yeux de Rolant: «La char oit blanche come nif desendue, / Color vermoil come graine vendue, / Boche petite, danteüre menue, / Oil oit riant, qant ert plus ireschue; / Sa blonde crine ne vos ai manteüe; / Soz ciel n'a home, tant ait chere barbue, / Ne la querist avoir en si braz nue" (L'Entrée d'Espagne, DXLII, v. 12555-12561). 
beauté, par son attitude pleine de dignité et de retenue ${ }^{15}$. L'apparition d'Emilia aux deux rivaux en amour Palemone et Arcita a lieu dans un locus amænus. Le narrateur s'arrête tantôt sur les effets produits par la beauté de la jeune fille sur les deux amoureux qui la regardent, tantôt sur la coquetterie de celle-ci qui se rend compte qu'elle a suscité l'admiration des deux jeunes hommes, détail, ce dernier, que ne pouvait manquer de relever le fin connaisseur de la psychologie féminine qu'est Boccace:

E parendole ciò saper per vero

D'esser piaciuta, seco si diletta,

E più se ne tien bella, e più s'adorna

Qualora poi a quel giardin ritorna ${ }^{16}$.

Dans les deux exemples cités, la description de la femme aimée se fait d'un point de vue subjectif, et cela n'est pas pour nous surprendre étant donné la dominante lyrique des deux poemetti, notamment de Filostrato.

L'apparition d'Angelica dans l'Inamoramento suit les clichés du récit chevaleresque en vers:

Essa sembrava matutina stella

E ziglio d'orto e rosa di verzeri;

In soma, a dir di lei la veritate,

Non fu veduta mai tanta beltade. (I, I, 21, 5-8)

Clichés, et ce n'est pas un détail négligeable, que Boccace n'avait pas méprisés non plus, comme le montrent ces vers du Teseida concernant Ippolita, la reine des Amazones:

Ipolita era a maraviglia bella

E di valore accesa nel coraggio;

Ella sembrava matutina stella

$\mathrm{O}$ fresca rosa del mese di maggio ${ }^{17}$.

Quels effets produit l'amour chez ceux qui en sont touchés? La passion, qu'elle soit naissante ou languissante, a de toute façon des conséquences néfastes sur ceux qui en sont victimes. C'est justement dans la

15. Filostrato, I, 27-28 et IV, 164-165 où Troiolo rappelle à Criseida: «Non mi sospinse ad amarti bellezza, / La quale spesso altrui suole irretire [...] ma gli atti tuoi altieri e signorili, / Il valore e 'l parlar cavalleresco, / I tuoi costumi più ch'altra gentili, / Ed il vezzoso tuo sdegno donnesco".

16. Teseida, III, 19, 5-8; pour la scène complète de l'apparition d'Emilia, ibid., strophes 5-19; une description très détaillée d'Emilia se trouve à la fin du poème: XII, 53-63.

17. Teseida, I, 125, 1-4; voir aussi "ell'è più chiara che alcuna stella» (Tes., IV, 35, 3); «bella vie più che gemma margherita» (Tes., IX, 49, 4). Sur les rapports de Boccace avec la production en ottava rima, voir Vittore BRANCA, Il Cantare trecentesco e il Boccaccio del Filostrato e del Teseida, Florence, Sansoni, 1936, notamment p. 15-18. 
représentation de la souffrance amoureuse que Boiardo pouvait trouver chez Boccace, notamment dans ses poemetti, plusieurs exemples susceptibles de l'intéresser. Mais pour mieux évaluer la dette de Boiardo à l'égard de Boccace dans la représentation de la souffrance amoureuse, il faut distinguer entre l'action principale de l'Inamoramento, qui a Orlando comme héros, et les digressions, pauses lyriques ou nouvelles. Dans Filostrato, œuvre lyrique illustrant comment un individu peut se faire détruire par la passion, l'amoureux se retire dans sa chambre pour pouvoir se jeter sur son lit et donner libre cours à son désespoir. Le motif de la chambre et du lit, récurrent dans Filostrato, symbolise l'impuissance du vaincu par l'amour qu'est Troiolo ${ }^{18}$. Celui-ci, seul dans sa chambre ( Standosi in cotal guisa un di'soletto / nella camera sua Troiol pensoso»), reçoit la visite de Pandaro, cousin de Criseida, à qui, honteux, il avoue les raisons de sa souffrance, après quoi il dissimule ses sanglots dans l'oreiller :

E sopra il letto ricadde supino,

Piangendo forte e nascondendo il viso. (Filostrato, II, 16, 1-2) ${ }^{19}$

Troiolo in Troia tristo ed angoscioso,

quanto fu mai nessun, se ne rivenne,

e nel viso fellone e niquitoso,

$[\ldots]$

ma se n'entrò in camera soletto (Filostrato, V, 15, 1-3, 8)

Orlando, de son côté, ayant su que son cousin Ranaldo, qui a, c'est bien connu, la réputation d'être un grand séducteur, est sur les traces d'Angelica, s'en va pleurer dans sa chambre:

Partisse in vista nequitosa e fella;

E sopra al letto suo cadde invilito. (Inamoramento, I, II, 22,4-5) ${ }^{20}$

E con lamenti e con sospir insani

Senza spogliarsi se gitò sul letto. (Inamoramento, I, XXV, 51, 5-6)

Cossì dicendo dal letto si leva,

Dove giaciuto avea sempre piangendo. (Inamoramento, I, II, 27, 1-2)

18. On n'insistera pas sur les dettes qu'autant Boccace que Boiardo ont à l'égard de Dante: le motif de la plainte dans la cameretta ayant été développé par ce dernier dans la Vita Nuova, voir R. Donnarumma, «Presenze...», cit., p. 581-582.

19. Voir aussi ces autres exemples, tous tirés de Filostrato: «e tal qual era sospiroso e pio, / [...] / nella camera ginne» (IV, 22, 3-6), "sopra il suo letto si gitto alquanto", "Erasi la dolente in sul suo letto / stesa gittata, piangendo sì forte» (IV, 87, 1-2); et cet autre exemple provenant de l'Inamoramento: "Gettasi al letto quella sconsolata / E giorno e notte di pianger non fina " (I, XII, 44, 3-4).

20. Voir aussi "Presto dette combiato ai due germani / E nela zambra se chiuse solleto" (Inamoramento, I, XXV, 51, 1-2). 
Il est important de souligner que cette représentation d'Orlando pleurant dans son lit ne concerne que le premier livre de l'Inamoramento, celui où l'intention parodique est déclarée. L'ironie naît du fait de mettre le redoutable guerrier insensible à l'amour de la tradition chevaleresque dans une situation de fragilité enfantine: "piangea nel letto come un vil garzone». Nous avons déjà constaté que Boiardo n'est pas le premier a avoir eu l'idée de mettre Orlando, et plus généralement les austères héros de la tradition carolingienne, dans des situations ridicules. Ce qui le distingue des autres est la finesse de sa touche et le lyrisme de Boccace lui offre de quoi satisfaire son penchant pour une légèreté élégante. Dans un roman français du début du XIII siècle, Girart de Vienne, pour ne citer qu'un exemple, Orlando, dans un raptus de passion, s'empare de la belle Aude et profite d'elle ${ }^{21}$. L'image que cet auteur donne d'Orlando frappe par sa cocasserie, mais ne nous fait pas sourire. Car au fond il n'y a pas un vrai décalage entre le guerrier de Charlemagne et la brute épaisse qui s'empare d'Aude, puisque sa force primaire, on pourrait dire sa virilité, n'est pas remise en cause. En revanche, Boiardo, en attribuant à Orlando les faiblesses d'un Troiolo, autrement dit en attribuant à un héros épique les traits d'un personnage lyrique, le renouvelle en profondeur en l'humanisant et en l'enrichissant. Ce n'est plus seulement du guerrier sans peur qu'il s'agit, et le courage d'Orlando n'est jamais remis en cause dans l'Inamoramento, mais aussi d'un homme sensible à la puissance de l'amour. Cette radicale transformation du personnage d'Orlando ne pouvait être réalisée que par le poète des Amorum Libri Tres, le chansonnier amoureux le plus important du Quattrocento, et du familier d'Isabella d'Este Gonzaga qui suivait avec impatience, on le sait, la progression du roman du comte de Scandiano.

Si la chambre est le lieu député aux plaintes amoureuses d'Orlando et de Troiolo, d'autres héros, autant de Boccace que de Boiardo, se lamentent dans un bosquet, c'est-à-dire dans un locus amoenus. Le choix de cet endroit offre au plaignant l'avantage de pouvoir être entendu par quelqu'un, souvent l'être aimé, ce qui est capital pour l'évolution de l'action. Ainsi Arcita, qui est revenu à Athènes en cachette et sous une fausse identité, est-il entendu par Panfilo alors qu'il se plaint dans un

21. «Rolland la prist molt bien à esguarder, / Dedans son cueur forment à gouleser. / Tant s ' $\mathrm{i}$ entant le vassaus adurés / Qu'il en oblie d'Olivier le jouster. / Le destrier point vers Aude, où est alés. Saisie l'a; qu'il l'en voloit porter / Et l'ost le Roi en son demoine tref. / Là en feist toute sa volonté», cité dans Françoise Théodore Annette VOIGT, Roland-Orlando dans l'épopée française et italienne, Leiden, Brill, 1938, p. 59-60. 
bosquet ${ }^{22}$; son ami et rival en amour Palemone en est vite informé, ce qui précipite les événements jusqu'au dénouement avec la mort d'Arcita et le mariage d'Emilia avec Palemone. De même, la plainte de Prasildo, toujours dans un bosquet, est entendue par Tisbina qui intervient en lui proposant une aventure impossible dans l'espoir de le détourner d'un amour qu'elle ne peut payer de retour étant déjà liée à Iroldo ${ }^{23}$.

Boccace revient dans ses œuvres sur les effets dévastateurs de la passion. Il évoque l'impossibilité de trouver le repos (Filostrato, V, 19, 1-2: «E sé in qua ed ora in là volgendo, / sanza luogo trovar per lo suo letto»), le dépérissement physique ("Aveagli già amore il sonno tolto, / E minuito il cibo, ed il pensiero / Multiplicato sì che già nel volto / Ne dava pallidezza segno vero") ${ }^{24}$, la tristesse ( «e come fuoco fuggiva 'l diletto, / ed ogni festa ed ogni compagnia» $)^{25}$, l'indécision des amoureux ${ }^{26}$. Ces aspects sont librement repris par Boiardo pour décrire les tourments de Prasildo, personnage de la nouvelle courtoise déjà évoquée, au chant XII du livre I:

Ma poi che il gioco è partito, e la festa,

Non parte già la fiama da il suo core,

Ma tuto il giorno intégro lo molesta,

La note lo assalisse in più furore.

Hora quella cagion ritrova, hor questa

Che al volto li è fugito ogni colore,

Che la quïete de il dormir gli è tolta

Né trova il loco, e ben spesso si volta. (Inamoramento, I, XII, 9)

La souffrance d'Orlando, en revanche, est décrite de façon très dramatique et colorée à travers les gestes qui rendent de manière expressive et vivante son malheur d'amoureux:

22. Teseida, IV, 63.

23. Inamoramento, I, XII, 26-28. Tisbina demande à Prasildo de lui rapporter une branche d'un arbre fabuleux, appelé arbre du trésor car ses branches sont en or et ses fruits sont des pierres précieuses: "In megio è un tronco in smisurata alteza / [...] / Che sempre perle getta nel fiorire, / Et è chiamato il Tronco de il Thesoro, / Che ha pomi di smiraldi e rami d'oro", ibid., I, XII, 27, 38. L'Orto di Medusa, ce jardin merveilleux où Prasildo doit se rendre sur ordre de Tisbina, est une libre adaptation du Jardin des Hespérides dans lequel poussent des arbres qui donnent des fruits d'or. Un jardin semblable se trouve aussi dans Filocolo de Boccace, IV, 85, 9.

24. Filostrato, I, 47, 1-4; voir aussi Teseida, XI, 46, 1-3: «E quinci rotti li tristi lamenti, / muta ricadde, / e il chiaro colore fuggì del viso"; Ibid., XII, 2, 1-3: «E del bel viso il vermiglio colore / s'era fuggito, e era diventata palida e magra", et aussi IV, 27, 1-3 et III, 34.

25. Filostrato, VII, 19, 6-7; de même Prasildo: «Non più festegia sì com'era usato : / In odio ha ogni dileto e ancor sé stesso" (Inamoramento, I, XII, 17, 1-2).

26. Decameron, $\mathrm{X}, 8,20:$ : $\mathrm{E}$ da questo ragionamento facendo beffe di se medesimo tornando in sul contrario, e di questo in quello e di quello in questo, non solamente quel giorno e la notte seguente consumò, ma più altri, in tanto che, il cibo e 'l sonno perdutone, per debolezza fu constretto a giacere ". Le motif du balancement entre deux choix opposés est évoqué aussi dans Teseida et dans Filostrato; l'indécision étant le propre du héros lyrique. 
Cossì, col cor di doglia tuto ardente,

Il Conte sieco stesso ragionava;

E quella notte non dormì nïente,

Ma spesso a ciascun lato si voltava. (Inamoramento, I, XXV, 59, 1-4)

Cossì dicendo dal letto si leva,

[...]

La sera aspecta (e lo aspetar lo agreva)

E su e giù si va tutto rodendo:

Uno atimo cento anni li rileva,

Hor questo adviso, hor quello in sé facendo. (Inamoramento, I, II, 27, 1-6)

Ce n'est pas que l'intention parodique, évidente, qui commande ce choix, mais les contraintes de l'oralité, pleinement assumée par Boiardo qui, tel un canterino, se préoccupe de faire revivre la scène sous les yeux de son public, d'où la nécessité de dramatiser le récit. On peut sourire, certes, de l'impatience d'Orlando, mais quiconque a connu les tourments de l'amour ressent une grande sympathie pour le pauvre Orlando. On peut dire qu'ici Boiardo sacrifie les motifs de la tradition lyrique au profit du réalisme et parvient ainsi à conquérir le cœur et l'esprit de son public qui s'amuse tout en éprouvant de la compassion pour le héros.

L'amour n'est cependant pas seulement une source de douleur, mais également d'élévation pour l'amoureux. Si dans un premier temps, celuici essaye de se faire remarquer par des marques extérieures, comme la tenue vestimentaire et le comportement ${ }^{27}$, il peut arriver, sur la demande de l'être aimé, à dépasser ses propres limites, car, comme il est dit dans l'Inamoramento, "la vertù crescie sempremai / Che se ritrova l'homo inamorato" (I, XII, 12, 3-4). Le motif de l'élévation par l'amour est au cour autant de l'œuvre de Boccace que de Boiardo et, chez ce dernier, se trouve illustré dans la nouvelle déjà mentionnée d'Iroldo, Prasildo et Tisbina, particulièrement significative pour nous car elle est inspirée des nouvelles V (Ansaldo et Madonna Dianora) et VIII (Tito et Gisippo) de la $\mathrm{X}^{\mathrm{e}}$ journée du Decameron ainsi que de Filocolo (IV ${ }^{\mathrm{e}}$ question du livre IV). On ne reviendra pas sur cette nouvelle, certes l'un des chapitres les mieux connus du poème de Boiardo et le lieu de l'Inamoramento où la présence de Boccace est la plus forte, quoique mêlée à d'autres suggestions, comme le motif du faux poison absorbé par Iroldo et Tisbina pour éviter la sépa-

27. Filostrato, II, 84, 1-3: «Troiolo canta e fa mirabil festa, / armeggia e dona e spende lietamente, / e spesso si rinnuova e cangia vesta»; Teseida, IV, 62, 1-6: " [Arcita] cantava e faceva gran festa; faceva pruove e vestia riccamente, / [...] / e' n fatti d'arme facea manifesta / la sua virtù "; Inamoramento, I, XII, 11, 6-8: Prasildo "Versi compone e canta in melodia, / Giostra sovente et entra a'torniamenti / Con gran destrieri e richi paramenti». 
ration. Ce motif provient, nous le savons, des Métamorphoses d'Apulée, ouvrage que Boiardo avait traduit. Mais, pour représenter la langueur et le désespoir qui saisit les amants avant l'absorption du breuvage, le comte de Scandiano se tourne à nouveau vers Boccace, plus précisément vers la très pathétique scène de la dernière rencontre entre Criseida et Troiolo:

E forte insieme amendue si stringieno

Di lagrime bagnati tutti quanti,

E volendo parlarsi non potieno,

Sì gl'impedivan gli angosciosi pianti. (Filostrato, IV, 115, 1-4)

Quei dui leal amanti e sventurati,

E col viso apogiato insieme stano,

Hor più che prima nel pianto afocati,

Né l'un dal'altro dipartir si sano. (Inamoramento, I, XII, 57, 2-5)

Mais de quoi se plaignent-ils ces amoureux? Le plus souvent, ils se plaignent de l'être aimé dont ils dénoncent l'indifférence par des formules typiques de la poésie lyrique qu'employaient déjà les héros de Boccace:

Cossì farò contenta quella altera

A cui la vita mia tanto dispiace,

Poi che ha voluto il ciel una alma fiera

Coprir in viso de pietose face.

Essa ha diletto che un suo servo piera. (Inamoramento, I, XII, 20, 1-5)

Tu che farai? Deh, dilmi, starai altera,

E lascerai colui, che sé non cura

Per amar te, a morte tanto fera

Venire? O reo distino, o rea ventura

Ch'un sì fatto uom per te amando pera! (Filostrato, II, 64, 1-5) 28

Même si dans Filostrato ce n'est pas l'amoureux qui prononce ces mots, mais Pandaro dans l'intention de faire plier Criseida, la dette à l'égard de Boccace est flagrante. Il ne faut toutefois pas oublier que ces mêmes expressions sont courantes dans la poésie amoureuse du $\mathrm{XV}^{\mathrm{e}}$ siècle, notamment dans le chansonnier de Boiardo, et on sait que ce dernier n'hésite pas à pratiquer l'auto-citation ${ }^{29}$.

Arcita et Orlando se plaignent pareillement de ne pouvoir oublier l'image de l'être aimé:

28. Sur ce même motif de l'impossibilité de l'image de la femme aimée, voir aussi Filocolo, III, 31,9 .

29. Voir par exemple ces vers tirés des Amorum Libri: «Viver voglio così, così morire, / poiché piace ad Amor che così viva, / e che così tra saxi amando pèra. / Quella crudel che la mia vita schiva / farà pur sazia la sua mente altera, / se parte del mio dol potrà sentire " (Matteo Maria BOIARDO, 
E non mi si di diparte della mente

L'imagine di quella creatura,

Né pensiero ho d'altra cosa niente;

Sì m’è fissa nel cor la sua figura,

E sì mi sta nell'animo piacente,

Ch'io mi riputerei somma ventura

S'io le piacessi com'ella mi piace;

E sanza ciò mai non credo aver pace. (Teseida, I, III, 21)

Io non mi posso dal cor dipartire

La dolce vista de il viso sereno. (Inamoramento, I, I, 31, 1-2)

Né sciò se quella angelica figura

Se dignarà de amar la mia persona ;

Che ben serà figliol dela Ventura,

E de felice portarà corona,

Se alcun fia amato da tal creatura! (Inamoramento, I, II, 24, 1-5)

Ils voient dans leur malchance amoureuse un effet secondaire, pour ainsi dire, du mauvais sort qui s'acharne contre eux depuis leur naissance. Ainsi Arcita:

- O misera Fortuna de' viventi,

Quanti dai moti spessi alle tue cose!

Deh, come abbassi li sangui e le genti. (Teseida, IV, 80, 1-3)

Di real sangue, lasso!, generato,

Venni nel mondo d'ogni pena ostello,

E con gran cura in ricchezza allevato,

Nella città di Bacco tapinello

Vissi e con gioia tenni grande stato,

Sanza pensare al tuo operar fello;

Poi per l'altrui peccato, non per mio,

La gioia e 'l regno e 'l sangue mio perio. (Teseida, IV, 81)

\section{Et Brandimarte:}

Ahi, Fortuna crudel, come a gran torto

Presa hai la guerra contro a' fatti miei! (Inamoramento, I, XXI, 44, 4-5)

[...]

Tolto m'hai del paese ove fui nato,

Ché ancor me odiasti essendo fanciullino;

Di mia casa reale io fui robato,

Canzoniere (Amorum Libri), éd. C. Micocci, Milan, Garzanti, 2006, p. 133), et ces autres provenant d'un chansonnier inédit du Quattrocento: «mercè chiamando sempre a quell'altera / che del mio mal sì fera / sempre si mostra e nuda de pietate, / und'el convien ch'io pera " Giannotto Calogrosso, Nicolosa bella. Prose e versi d'amore del secolo XV, éd. F. Gaeta / R. Spongano, Bologne, Commissione per i testi di lingua, 1959, LXXXVI). 
E venduto per schiavo piccolino;

Il nome de mio patre agio scordato

E il mio paese, misero! tapino! (Inamoramento, I, XXI, 45, 1-6)

Ces lamentations débutent toutes les deux par une apostrophe à la fortune. Sauf qu'Arcita a l'attitude passive de la victime, alors que Brandimarte accuse la fortune de le persécuter à tort. En ce qui concerne les différences, nous pouvons observer que le malheur d'Arcita succède au bonheur par une sorte de fatalité extérieure ("per l'altrui peccato»), alors que Brandimarte doit ses infortunes à un fait très précis, celui d'avoir été enlevé tout petit. Il s'agit d'une péripétie courante de la comédie latine. Nous savons en effet que l'histoire de Brandimarte est inspirée par les Captivi de Plaute, et que les péripéties ici rapidement anticipées par le plaignant seront dûment développées dans les chants XII et XIII du livre II consacrés à la découverte des origines de Brandimarte, personnage central de l'Inamoramento, incarnation du parfait chevalier, courageux et courtois malgré sa naissance païenne ${ }^{30}$. La mise en parallèle de ces deux plaintes illustre bien l'art dans lequel Boiardo est passé maître, c'est-à-dire l'habileté à mêler et à fondre harmonieusement dans sa propre œuvre les sources les plus disparates provenant de la tradition médiévale et antique.

Que font les amoureux pour obtenir les faveurs de la femme aimée? Arcita s'enfuit de la prison où il est enfermé pour avoir plus de chances de conquérir Emilia. Mais, incapable de supporter cet éloignement, il revient sous une fausse identité et accepte de se battre ouvertement contre son rival: le vainqueur du grand tournoi organisé par Thésée aura Emilia ${ }^{31}$. Il y a quelque chose de velléitaire et de raté dans ce départ d'Arcita, car sa quête est sans but. Il ne va ni à la recherche de la femme aimée, qu'il laisse près de son rival, ni accomplir une mission qu'elle lui aurait confiée. Victime de la malveillance des dieux, Arcita incarne le héros lyrique qui n'a pas de véritable marge d'action, semblable en cela à Troiolo.

Orlando aussi décide de partir, mais avec un but précis car il va sur les traces d'Angelica qui se dirige vers son fabuleux royaume asiatique. Même si Orlando finit par revenir au point de départ, ce n'est pas parce qu'il a échoué dans sa quête comme Arcita, car il emmène Angelica avec lui. C'est le fonctionnement du roman chevaleresque qui impose le retour du héros de la périphérie romanesque, le Cathay où réside Angelica, au centre épique, la France de Charlemagne. C'est dans l'Entrée d'Espagne que, pour

30. L'adaptation romanesque des Captivi de Plaute par Boiardo a été étudiée par Marco Villoresi, Da Guarino a Boiardo. La cultura teatrale a Ferrara nel Quattrocento, Rome, Bulzoni, 1994, p. 133-149. 
la première fois dans un roman carolingien, Orlando quitte la France pour l'Orient. Ce choix, déterminant pour l'évolution du roman chevaleresque, ouvre la voie aux aventures romanesques d'Orlando chez les infidèles et donc à la fusion entre le courant épique et le courant romanesque.

Le départ d'Orlando dans l'Inamoramento se déroule selon un cérémonial bien précis: pendant la nuit, en cachette, sous une fausse identité:

Ma come gionta fo la notte scura,

Nascosamente veste l'armadura. (I, II, 27, 7-8)

Già non portò la insigna de il quartiero,

Ma de un vermiglio scuro era vestito;

Cavalca Brigliadoro, il cavaliero,

E soletto ala porta se n'è gito.

Non scià de lui famiglio né scudero:

Tacitamente è dela terra usito. (I, II, 28, 1-6)

Ce rituel est courant dans la tradition italienne. On en rencontre des antécédents dans l'Entrée d'Espagne et dans la Spagna F, d'auteur inconnu, dont le manuscrit est conservé à la Biblioteca ariostea de Ferrare. Ici aussi se trouvent les éléments de l'obscurité et du déguisement ${ }^{32}$.

À côté de ces amoureux qui se débrouillent tout seuls, il y en d'autres qui s'adressent à un intermédiaire pour essayer de faire fléchir l'être aimé. Troiolo fait appel à Pandaro pour convaincre la rétive Criseida et Prasildo s'adresse à une amie de Tisbina. Mais si le héros romanesque qu'est Prasildo s'engage dans une aventure apparemment impossible par amour de Tisbina, aventure qui lui réussit, car comme le dit Boiardo, rien n'est impossible à l'homme amoureux ${ }^{33}$, Troiolo, en revanche, trouve toujours une bonne excuse pour ne pas agir. Il pense un instant se déguiser en pèlerin pour aller chercher Criseida chez les Grecs, mais il repousse aussitôt cette idée sous un prétexte bien peu convaincant:

Ma sì non si sapeva contraffare

Che gli paresse assai coprire il vero,

Né scusa degna sapeva trovare

Da dir, se fosse stato conosciuto

In abito cotanto disparuto. (Filostrato, VIII, 4, 4-8)

31. Charlemagne aussi promet de donner Angelica à celui qui, entre Orlando et Ranaldo, s'illustrera le plus sur le champ de bataille.

32. "Mezanote era quando se partia " (Spagna F XII, 31), "La sopravesta a quartier che solìa / portar in ogni parte dove andava / una con un lion se ne vestì / e quella dentro al padiglion lassava" (ibid., XII, 30), in Inamoramento, ed. cit, vol. I, p. 67, notes 27 et 28.

33. Inamoramento, I, XII, 12, 3-4: «La vertù crescie sempremai / Che se ritrova l'homo inamorato». 
Nous touchons encore une fois à la différence substantielle existant entre le héros romanesque, tourné vers l'aventure et le mouvement, et le héros lyrique qui n'agit pas, mais nous dévoile son cœur.

Les intermédiaires chargés, autant par Troiolo que par Prasildo, de faire capituler la femme aimée, font appel à des topoi bien connus. Pandaro, après avoir souligné les qualités et la haute position sociale de l'amoureux, ce que fait aussi l'émissaire de Prasildo auprès de Tisbina ${ }^{34}$, invite Criseida à saisir la belle occasion qui se présente à elle, car la jeunesse ne dure pas éternellement:

Solo una volta ha nel mondo ventura

Qualunque vive, s'ei la sa pigliare;

Chi lei vegnente lascia, sua sciagura

Pianga da sé sanza altrui biasimare. (Filostrato, II, 44, 1-4)

Cette même idée, quoique différemment exprimée, revient aussi sous la plume de Boiardo:

Se ala Ventura non dati de piglio,

Ela si turba e vòltavi la facia.

[...]

A che da voi Fortuna è biastemata?

Ché la colpa è di lei, ma il danno è vostro ${ }^{35}$.

La situation n'est toutefois pas la même, car ces mots sont prononcés à l'intention d'Orlando lorsqu'il est aux prises avec Morgana, qui incarne la Fortuna dans le très allégorique chant IX du livre II. Aux caprices du sort, Orlando, héros romanesque conçu par un auteur profondément humaniste, c'est-à-dire ayant confiance en l'homme, comme le comte de Scandiano, répond par la patience et la détermination, alors que ni Troiolo ni Arcita, pour des raisons différentes, ne parviennent à résister aux coups du mauvais sort. L'invitation à saisir l'occasion s'accompagne d'une invitation au carpe diem surtout à l'intention de la dame:

Non perder tempo, pensa che vecchiezza

$\mathrm{O}$ morte torrà via la tua bellezza ${ }^{36}$.

34. Filostrato, II, 42, 1-6: «Egli è d'animo altiero e di legnaggio / onesto molto, e cupido d'onore, / di senno natural più ch'altro saggio, / né di scienza n'è alcun maggiore ; / prode ed ardito e chiaro nel visaggio, / io non potrei dir tutto il suo valore»; Filostrato, II, 76, 1-3: "[...] questi ch'al presente t'ama / è di troppo più alta condizione / che tu non sei »; Inamoramento, I, XII, 14, $1-4:$ « [... " "O bella dama, / Cognosci l'hora dela tua ventura, / Dapoi che un tal baron più che sé te ama, / Che non ha il ciel più vaga creatura" ".

35. Inamoramento, II, IX, 1, 3-4 et 2, 1-2; voir aussi II, VIII, 44.

36. Filostrato, II, 54, 7-8. Voir aussi, toujours dans Filostrato: «La giovinezza mia si fugge ogni ora, / debbol'io perder sì miseramente?» (II, 70, 1-2); «Chi mi vorrà se io c'invecchio mai ? / Certo nessuno [...] / Buon è adunque a tempo provvedersi» (II, 71, 1-6). 
Prende diletto mentre sei su il verde,

Che lo aùto piacer mai non se perde! (Inamoramento, I, XII, 14, 7-8)

À peine évoqué dans la nouvelle d'Iroldo, Tisbina et Prasildo, cet argument connaît en revanche un long développement dans Filostrato où Criseida analyse attentivement le pour et le contre avant de céder aux pressions de Troiolo.

La récompense qu'attend l'amoureux après tant de souffrances et des prières est bien évidemment la jouissance amoureuse. Boiardo, tout compte fait, accorde peu de place à la représentation de cette jouissance, à quelques remarquables exceptions près. Les ébats entre Brandimarte et Fiordelisa, le seul couple d'amoureux comblés du roman, sont décrits avec réalisme et délicatesse à la fois :

Stavan sì streti quei dui amanti insieme

Che l'aria non potrebe tra lor gire

[...]

Come ciascun sospira e ciascun geme

De alta dolceza non saprebe io dire.

Parve niente a loro il primo gioco,

[...]

E nel secondo assalto intrarno al loco

Che al primo ascontro apena fu tocato,

Sospirando de amore, e a poco a poco

Se fu ciascun di loro abandonato

Sei volte ritornarno a quel danzare

Prima che il lor desir ben fosse spento ${ }^{37}$.

Boiardo peut être encore plus explicite en laissant directement la parole aux amants qui relatent leurs sensations de plaisir, comme le fait Leodilla, l'héroïne de l'une des sept nouvelles de l'Inamoramento ${ }^{38}$. Cette nouvelle, concernant l'astuce d'une épouse et de son jeune amant pour se débarrasser d'un mari jaloux, âgé, impuissant et pervers, illustre encore une fois l'habileté de Boiardo à tirer parti des sources les plus variées. Le motif de la nouvelle fait tout de suite venir à l'esprit la septième journée du Decameron consacrée aux mauvais tours joués à des maris jaloux par leurs femmes rusées et actives ${ }^{39}$. Comme d'habitude, Boccace n'est pas l'unique

37. Inamoramento, I, XIX, 61, 1-2, 5-6; 62, 1, 3-6; 63, 1-2. Après les ébats, les amants s'endorment et Fiordelisa est enlevée par un lubrique homme sauvage, suggestion que Boiardo a tirée de Gismirante d'Antonio Pucci (I, 44-45), voir R. Donnarumma, Storia dall'Orlando innamorato, cit., p. 108.

38. Voir Inamoramento, I, XXII, 26.

39. Voir notamment les nouvelles 5 et 8 . 
source, car Boiardo regarde également du côté des Anciens, plus précisément du côté des Métamorphoses d'Ovide ainsi que du Miles gloriosus de Plaute. L'auteur de l'Inamoramento reprend ainsi d'Ovide le mythe d'Atalante et du Miles gloriosus le motif des fausses jumelles. Il les réélabore et réinterprète en fonction de ses exigences, c'est-à-dire en les réadaptant au climat un peu trouble et inquiétant, quand il n'est pas chargé d'horreur, de ses nouvelles. Celles-ci sont de véritables moments forts visant à l'illustration des méfaits de la passion amoureuse portée à ses conséquences extrêmes, la jalousie du vieux Folderico dont il est question dans la nouvelle de Leodilla allant jusqu'à la maladie.

Mais revenons aux deux derniers vers cités plus haut par lesquels se termine la description des ébats de Brandimarte et Fiordelisa. Pour ceux-ci, Boiardo s'est souvenu du récit de la première nuit entre Emilia et Palemone au sujet de laquelle il est dit: "Venere, anzi che 'l dì fosse chiaro, / sette volte raccesa e tante spenta / [fu] nel fonte amoroso" (Teseida, XII, 77, 3-5). Mais l'épisode des retrouvailles érotiques entre Brandimarte et Fiordelisa doit également beaucoup à Filostrato, notamment à la première rencontre entre Troiolo et Criseida, dont Boiardo a pu $s^{\prime}$ inspirer ${ }^{40}$. Car Troiolo, avant d'être séparé de son amie, a pu jouir de ses faveurs. On comprend mieux alors qu'il ne sache pas se résigner à avoir été oublié d'elle et qu'il rétorque à Pandaro que la perte d'un bien est plus dure à accepter que de n'avoir jamais rien eu : «quasi ragion che men pena sia / il perder che il non aver niente» (Filostrato, IV, 56, 2-3). On retrouve la même considération, à quelques détails expressifs près, dans la bouche d'Orlando: "che perder l'acquistato è maggior doglia / che il non acquistar quel de che s'ha voglia» (Inamoramento, I, XXV, 53, 7-8). Les conclusions des deux amants éconduits sont les mêmes, mais pas la situation. Troiolo vient de perdre un bien dont il a joui, alors qu'Orlando non seulement ne pourra jamais posséder Angelica, pire encore il est lui-même le premier responsable de cette frustrante condition. Arrivé à Albraca, où Angelica s'est réfugiée, il est chaleureusement accueilli par celle-ci qui l'embrasse, le déshabille et lui donne personnellement un bain sans qu'il essaie même de tirer profit de la situation. Il prononce alors les mots de dépit précédemment évoqués en apprenant que son rival Ranaldo, notoire séducteur, se trouve également auprès d'Angelica. Le narrateur ironise donc sur le fait qu'Orlando s'en prenne à son mauvais sort et à Ranaldo,

40. Voir Filostrato, III, 29-35, où on peut notamment lire ces vers: «Ei si spogliaro ed entraron nel letto» (31, 4), «E strignendo l'un l'altro con fervore, / d'amor sentiron l'ultimo valore» (32, 78). 
alors que c'est d'abord sa timidité qui l'empêche de tirer profit d'Angelica pratiquement offerte à lui. Et l'ironie est double si l'on pense qu'Orlando redoute le séducteur qu'est Ranaldo quand celui-ci n'en est plus un. S'étant abreuvé à la source de la Haine au début du livre I, Ranaldo a perdu tout intérêt pour la chose amoureuse, intérêt qu'il ne pourra récupérer que bien plus tard au chant XV du livre II. Rappelons que pour la scène du bain et du massage, Boiardo est encore débiteur de Boccace, notamment d'une nouvelle du Decameron (VIII, 10). Dans celle-ci, Salabaetto, un marchand toscan, est lui aussi massé, lavé et caressé par une Sicilienne malintentionnée qui n'en veut qu'à ses biens. Salabaetto, quoique trompé, tire toutefois sexuellement profit de la situation, ce que ne sait pas faire Orlando. Nous avons là un exemple particulièrement heureux de l'adaptation d'un motif propre à la nouvelle vers le roman chevaleresque, dont Boiardo a enrichi et dilaté la capacité naturelle à accueillir les suggestions des provenances les plus diversifiées.

En proclamant la supériorité de la matière arthurienne sur la matière carolingienne (cf. I, XVIII, exorde) et en soulignant la puissance de l'amour, Boiardo a également fait plus de place dans son roman aux personnages féminins, il suffit de citer en exemple Angelica. Il n'en reste pas moins que comme dans tout roman chevaleresque qui se respecte les valeurs dominantes sont masculines. On célèbre la force, le courage, la loyauté, la générosité, la courtoisie, qualités que partagent tous les héros de l'Inamoramento, mais non les héroïnes. À leur égard, nous retrouvons sous la plume de Boiardo les arguments de la misogynie traditionnelle, issue ou pas du roman chevaleresque. Même dans ce domaine-ci, Boccace a fourni au comte de Scandiano des suggestions qu'il a su retenir. L'idée de la volubilité et de la légèreté des femmes, incapables de sentiments profonds et durables, est partagée par les deux auteurs. Nous la retrouvons ainsi en guise de morale à la fin de certains de leurs récits: "Ciascuna dama è molle e tenerina, / Cossì del corpo comme dela mente", lit-on à la fin de la nouvelle d'Iroldo, Tisbina et Prasildo pour justifier le revirement de Tisbina qui, amoureuse d'Iroldo, se tourne vers Prasildo ${ }^{41}$.

Pour décrire la volubilité féminine, incarnée sous sa forme extrême, il est vrai, par la coquette perverse qu'est Origille, emblème parfait de la male pucele des romans arthuriens, Boiardo recourt à l'image de la feuille secouée par le vent: "Come al vento si volge hor per sì stessa, / Cossì sempre rivolse ogni promessa» (I, XXIX, 2, 7-8), image déjà présente dans

41. Nous rappelons qu'Emilia est donnée par Thésée à Palemone, tout comme Sofronia est donnée à Tito. 
Filostrato: "Che è a porre in donna alcuno amore? / Ché come al vento si volge la foglia ${ }^{42}$ ». Il s' agit toutefois d'un topos littéraire trop répandu pour pouvoir en indiquer l'origine précise ${ }^{43}$. Il faut également signaler la différence du contexte. Chez Boccace, la comparaison entre la femme et la feuille est une simple observation de Pandaro afin de consoler Troiolo ou bien un constat du narrateur; chez Boiardo, elle est prononcée par un amoureux, victime de la méchanceté de la dame, à laquelle il a infligé pour la punir un supplice dantesque, car elle est suspendue à un arbre par les cheveux et se balance comme une feuille agitée par le vent. L’idée de la légèreté féminine se traduit donc ici dans une image autrement percutante que chez Boccace.

Dans un cas comme dans l'autre, la femme n'est pas douée de volonté propre, mais est une marchandise d'échange, au mieux un prétexte pour valoriser la grandeur d'âme des héros. C'est la morale émergeant des nouvelles de Madonna Dianora, de Tito et Gisippo, de Teseida ainsi que de la nouvelle d'Iroldo, Prasildo et Tisbina. Dans le pire des cas, elle est légère et insensible, comme Criseida qui oublie vite Troiolo, flattée par la cour de Diomède ou comme Origille, emblème de la femme maléfique selon la tradition romanesque. La volubilité des héroïnes de Boiardo, à la différence de celles de Boccace, procède davantage des nécessités narratives que de leur nature. Ainsi le jugement rapide émis sur Tisbina est fortement motivé par l'exigence de couper court à un récit qui a trop duré encore plus que par un penchant misogyne du narrateur. Par rapport à Boccace, la misogynie chez Boiardo tient plus d'un jeu expressif et narratif, visant à agrémenter le récit et à amuser le public, que d'une réflexion sérieuse sur le statut féminin. Qu'il s'agisse d'hommes ou de femmes, le narrateur de l'Inamoramento manifeste une certaine sympathie à l'égard des faiblesses humaines de ses personnages tant qu'elles restent dans des limites raisonnables, alors qu'il les condamne sans appel lorsqu'elles sombrent dans l'excès, ce qui arrive dans certaines nouvelles du roman où la cruauté n'a, pour ainsi dire, pas de sexe.

C'est au niveau de l'épanchement lyrique de la souffrance amoureuse que l'influence de Boccace sur Boiardo se fait le plus sentir. C'est surtout dans les deux poemetti, et notamment dans Filostrato, que Boiardo pouvait

42. Filostrato, I, 22, 1-2; l'idée de la volubilté féminine revient aussi à la fin de Filostrato en guise de mise en garde du narrateur à l'intention des jeunes amants: "Giovane donna, e mobile e vogliosa / È negli amanti molti» (VIII, 30, 1-2).

43. Il a été utilisé par Ovide (Hérö̈des, V, 109-110), par Calpurne (III, 10) et aussi par Pétrarque «Femina è cosa mobil per natura» ( $R V F$, CLXXXIII, 12); voir, Inamoramento, ed, cit., vol. I, p. 777, note 2 . 
trouver les suggestions les plus utiles à l'expression de l'intériorité, domaine dans lequel Boccace est passé maître. L'influence de Boccace est aussi évidente sur les nouvelles du poème, mais elle n'est pas exclusive, car à ce niveau-ci est également forte celle des Anciens et de la tradition arthurienne; c'est peut-être cette contamination de traditions si disparates qui confere un caractère très inquiétant aux nouvelles de l'Inamoramento.

\section{Les armes}

En ce qui concerne la matière guerrière, nous commencerons par examiner le fameux épisode du duel entre Orlando et Agricane (Inamoramento, I, XVIII-XIX), épisode qui, comme l'a montré Raffaele Donnarumma, a été amplement influencé par le combat entre Arcita et Palemone dans le livre V de Teseida ${ }^{44}$.

Pour bien analyser le récit de l'affrontement Orlando-Agricane et faire le point sur ses dettes à l'égard de Teseida ainsi que d'autres sources, il convient de le diviser en trois parties comprenant les circonstances et le lieu du duel, la pause nocturne et la conversation entre les deux guerriers et enfin l'épilogue avec la mort et le baptême d'Agricane. Rappelons d'abord les circonstances du duel. Orlando et Agricane, chrétien l'un, païen l'autre, sont en train de se battre depuis longtemps lorsque la nuit les surprend; ils décident alors de se reposer dans une prairie près d'une fontaine et d'y attendre l'aube pour reprendre leur affrontement. Dans la Spagna $F$, qui est le modèle chevaleresque le plus proche de ce combat, Orlando et Ferraù s'arrêtent eux aussi à la tombée de la nuit mais se séparent pour regagner leurs camps respectifs avec la promesse de se retrouver le lendemain ${ }^{45}$. L'idée de faire se reposer côte à côte deux guerriers enne-

44. R. Donnarumma, «Presenze...», cit., p. 553.

45. Il Combattimento di Orlando e Ferraù, I, 7-8, in La Spagna, poema cavalleresco del secolo XIV edito e illustrato da Michele Catalano, Bologne, Commissione per i testi li lingua, 1939, 3 vol. Pour la complexe histoire de la datation et de la composition de la Spagna in rima, on se reportera à l'introduction, toujours fondamentale, de M. Catalano (vol. I), avec les correctifs apportés par Carlo DionisotTi, "Entrée d'Espagne, Spagna, Rotta di Roncisvalle», in Boiardo e altri studi cavallereschi, éd. G. Anceschi - A. Tissoni Benvenuti, Novare, Interlinea, 2003, p. 15-50 (déjà in Studi in onore di A. Monteverdi, Modène, 1959, p. 207-241). Dionisotti, à la différence de Catalano, ne pense pas que Il Combattimento di Orlando et Ferraù soit un ouvrage autonome par rapport à la Spagna, mais qu'il témoigne simplement de l'existence de deux version du même poème, l'une plus longue en 40 cantari (Spagna maggiore), l'autre plus brève en 36 cantari (Spagna minore), tradition à laquelle remonte aussi le manuscrit conservé à la Biblioteca Ariostea de Ferrare (la Spagna F) et qui appartenait au duc Borso; voir Antonio FrANCESCHETTI, «La Spagna in rima e il duello di Orlando e Agricane", Lettere Italiane, XXI, 1969, p. 322-326, p. 323. Pour une mise 
mis n'est pas une invention de Boiardo et Pio Rajna est à même d'en citer d'autres exemples ${ }^{46}$, mais seul le comte de Scandiano développe ce détail avec art et humanité.

Le lieu où se déroulent les duels est un locus amønus chez Boccace, une "selva piana» non mieux caractérisée dans l'Inamoramento, sinon par la présence d'une fontaine en pierre, topos du roman arthurien. Chez Boccace, rien en principe ne laisse prévoir que l'endroit calme et beau où Arcita va se reposer et se plaindre à l'écart du monde, deviendra un lieu de combat ${ }^{47}$. Et en effet, c'est par hasard que les deux amis s'affrontent ici, alors qu'Orlando et Agricane, déjà en train de se battre, sont surpris par la nuit dans un lieu qui se prête à une trêve des armes. Le lieu de l'affrontement est sobrement décrit chez Boiardo et l'accent est mis sur la fontaine, élément récurrent du roman chevaleresque aux significations multiples. Dans le cas spécifique, elle a une valeur religieuse, car c'est d'elle qu'Orlando puise l'eau pour baptiser Agricane mourant. Dans la Spagna $F$, le combat entre Orlando et Ferraù se déroule sur un pont, autre lieu symbolique de la littérature chevaleresque, ici lieu de passage entre la vie et la mort ${ }^{48}$. Dans l'Inamoramento, l'espace du duel a toutes les caractéristiques du lieu théâtral, c'est-à-dire d'un espace sommairement décrit qui tire son sens de l'action tragique qui va bientôt s'y dérouler.

La conversation nocturne entre Orlando et Agricane comporte deux phases. Pendant la première, les deux guerriers exposent leurs convictions religieuses ainsi que leurs idées de la perfection chevaleresque. En ce qui concerne l'élément religieux, Boiardo amplifie et développe une simple suggestion de la Spagna F. Durant l'affrontement, Ferraù demande au champion chrétien des preuves de l'existence de son Dieu et celui-ci lui indique la nature environnante: "Ma credi tu in colui ch'è luce vera, / Che fece celo e terra, mane e sera ${ }^{49}$. L'Orlando de Boiardo recourt aux mêmes arguments mais mieux développés et exposés:

au point récente de la question, voir La Spagna in rima del manoscritto comense, éd. G.B. Rosiello, Alessandria, Edizioni dell'Orso, 2001, p. 17-20.

46. Voir Inamoramento, ed. cit., vol. I, p. 529, note 39. Dans l'Entrée d'Espagne, Orlando met une pierre sous la tête de son rival endormi pour qu'il puisse mieux reposer (CXLIII, v. 35353541).

47. Teseida, V, 34, 1-4: «E poi che [Palemone] fu di sopra la rivera / Sotto il bel pino infra le fresche erbette / Che li'avea produtte primavera, / Vide dormire Arcita»; cette description rappelle un autre fameux locus amoenus, celui où coule la rivière de l'amour près de laquelle s'endort Ranaldo: «Tutti li fior che mostra primavera / Avea quivi dipinto la Natura / E faceano ombra sopra a quella riva / Un fagio, un pino et una verda oliva" (Inamoramento, I, III, 37, 7-8).

48. Combattimento, I, 38, 1-5: «Feraù disse a lui :- Tu m'hai contento. / Or vore'io che noi ne renchiudessemo / in su quel ponte, se 'l t'è in piacimento, / ed ive insieme su tanto ci dessemo / che l'uno avesse l'altro a suo talento. - $\gg$.

49. Combattimento, I, 29, 7-8. 
- Questo che ora vediamo è un bel lavoro

Che fece la divina monarchia:

E la luna de argento e 'stele d'oro

E la luce del giorno e il sol lucente;

Dio tuto ha fato per la humana gente -. (Inamoramento, I, XVIII, 41, 3-8)

Ferraù, irrité, coupe court à ces discours et invite Orlando à reprendre le $\mathrm{combat}^{50}$. Agricane, en revanche, après avoir rapidement liquidé l'argument religieux et avoué son peu de goût pour les études, alors qu'Orlando en fait l'apologie, invite finalement son rival à lui parler de ses amours ${ }^{51}$. C'est là est en effet le seul sujet sur lequel - pensent-ils - deux chevaliers ennemis peuvent tomber d'accord. Mais nous savons que, loin d'être une source d'apaisement, l'amour qu'ils se découvrent pour la même femme, Angelica, réveille à nouveau leur inimitié et déclenche l'issue fatale pour Agricane. Cet élément offre une belle illustration de l'habileté de Boiardo dans le maniement de l'ironie: source de rire, mais également de drame.

C'est à partir du moment de la découverte de leur rivalité que l'influence du livre $\mathrm{V}$ de Teseida se fait déterminante sur cet épisode, alors que la Spagna $F$ n'offre aucune suggestion à ce sujet, puisque il n'est jamais question de rivalité amoureuse dans l'affrontement qui oppose Orlando à Ferraù. Agricane demande à Orlando de renoncer à Angelica:

Hor de una cosa te voglio pregare;

Che prima che vegniamo a cotal piato,

Quella dongiella che il tuo cor desìa

Tu l'abandoni e làsila per mia. (Inamoramento, I, XVIII, 50, 5-8)

Sa requête reprend aux termes près, comme nous pouvons le voir, celle de Palemone à Arcita afin qu'il accepte de lui laisser Emilia :

E tu so ch'ancor l'ami similmente,

Ma più che d'uno ella esser non poria

Per ch'io ti priego molto caramente

Che tu consenta che ella sia mia. (Teseida, V, 40, 1-4)

Orlando refuse, bien évidemment, de renoncer à Angelica: «Ma si quel che hor mi chiedi io prometesse, / E se io il giurasse, io non lo atenderei»

50. Combattimento, I, 31, 1-3: «Feraù disse: - El tuo parlar è ciancia./ Prendian del campo e ciascun s'acomandi / a lo suo dio che 'l scampi de rea mancia -».

51. "Perché ogni cavalier ch'è sanza amore / Se in vista è vivo, vivo è sanza core!» (Inamoramento, I, XVIII, 46, 7-8). Cette fameuse formule se trouve aussi à la fin du premier sonnet des Amorum Libri; il s'agit d'une formulation très ancienne qui remonte à Bernart de Ventadorn: «Ben es mortz qui d'amor non sen / al cor cal que dousa sabor" (Non es meravelha s'eu chan, v. 9-10); voir Inamoramento, ed. cit., vol. I, p. 532, note 46. 
(I, XVIII, 52, 3-4), tout comme Arcita avait opposé une fin de non recevoir à Palemone lui demandait de ne pas aimer Emilia : «E se io qui con fé ti promettessi / Di non amarla, credi tu che fare / Con tutto il mio ingegno io il potessi ?" (Teseida, V, 51, 1-3). L'absurdité de cette hypothèse est illustrée dans les deux cas par le recours à l'adynaton, la figure rhétorique de l'impossibilité:

Certo più tosto sanza mai mangiare

Crederei viver che d'amarla stessi;

E amor non si può così cacciare

Come tu credi; e poco ama chi posa,

Per impromessa, d'amare una cosa. (Teseida, V, 51, 4-8)

Cossì porìa spicar mie membre istesse

E levarme di fronte li ochi mei,

E viver sanza spirto e sanza core,

Come lasciar de Angelica lo amore! (Inamoramento, I, XVIII, 52, 5-8)

Les deux personnages partagent le même point de vue, mais comme il a été justement remarqué par la critique, le ton chez Boccacce est beaucoup plus quotidien et bourgeois que chez Boiardo où la tonalité dominante est celle de la courtoisie chevaleresque ${ }^{52}$. Dans l'Inamoramento, au refus d'Orlando de renoncer à Angelica fait immédiatement suite la reprise du duel, alors que dans Teseida l'affrontement est retardé par une parenthèse mythologique où Arcita rappelle les nombreuses persécutions divines dont sa race a fait l'objet. C'est-là l'occasion pour Boccace d'étaler son érudition, ainsi que de rappeler la malédiction qui pèse sur la race d'Edipe dont descend Arcita, le modèle de Teseida étant la Thébaïde de Stace. Avant de reprendre le combat, Agricane exprime son regret de donner la mort à un adversaire aussi valeureux qu'Orlando :

Se pur te piace meco battagliare,

Morto ne rimarai su questo campo,

Ma siami testimonio il ciel e il sole

Che darti morte mi dispiace e dole. (Inamoramento, I, XVIII, 35, 5-8)

Ce qui fait écho aux paroles qu'Arcita adresse à Palemone avant de commencer leur duel fratricide:

Ma siemi il ciel, che queste cose vede,

Ver testimonio, e Appollo surgente

E 'Fauni e le Driadi, se si crede

$[\ldots]$

Come io son del combatter dolente. (Teseida, V, 62, 1-6)

52. Voir Inamoramento, vol. I, p. 534, note 52. 
Dans la Spagna F, les deux ennemis, tout en reconnaissant leur valeur réciproque, ne sont pas touchés par de telles pensées; l'un et l'autre formulent le vou d'abattre l'ennemi pour faire cadeau d'un tel exploit à leurs dieux respectifs ${ }^{53}$. Si cette piété à l'égard de l'adversaire se comprend dans Teseida où s'affrontent deux amis, la circonstance est absolument remarquable chez Boiardo, les combattants étant deux ennemis appartenant à des horizons culturels et religieux éloignés.

La Spagna $F$ redevient le modèle de référence pour la description de l'issue du duel entre Orlando et Agricane, avec toutefois quelques clins d'œil à Teseida. Autant Ferraù qu'Agricane, mourant, reconnaissent la supériorité de la foi chrétienne et demandent le baptême ${ }^{54}$. Mais la délicatesse et la piété dont fait preuve Orlando après avoir blessé à mort son adversaire doit quelque chose au Teseida. Tant Arcita qu'Orlando retirent doucement les blessés de leurs montures, le premier par une décision spontanée, Palemone étant évanoui, le deuxième à la demande expresse d'Agricane: "Elli il tirava degli arcion di fori / Soavemente, e l'elmo li traeva"; "Io non me posso ormai più sostenire / Levame tu de arcion, baron accorto ${ }^{55}$ ». Ils les déposent ensuite dans un lieu de repos ( $\mathrm{E}$ ' $\mathrm{n}$ su l'erbetta fresca e sopra i fiori / Teneramente a giacer lo poneva»; "Ricolse il re ferito nelle braccia, / E sopra al marmo il pose alla fontana ${ }^{56}$ ). Orlando baptise Agricane avec l'eau de la fontaine; ses gestes ne sont pas décrits, mais on les imagine plus proches de ceux d'Arcita essayant de ranimer Palemone que de ceux de son homologue de la Spagna F. Pour ranimer son ami, Arcita puise de l'eau dans la rivière d'à côté: "E 'l viso li bagnava accio'che esso, / Se fosse vivo, si sentisse addesso » (Teseida, V, 69, 7-8); dans la Spagna F, Orlando jette en revanche l'eau un peu brutalement sur la tête de son rival après lui avoir retiré son casque: "Traseglie l'elmo e gitogliela in testa / E così batezò el baron perfetto" (Combattimento, II, 9, 2-3). Dans la Spagna F il n'y a aucune allusion au chagrin d'Orlando, alors que Boiardo parle explicitement des larmes d'Orlando, de son repentir et de ses prières pour le salut de son noble ennemi :

53. Combattimento, I, 4, 5-8: «Diceva Orlando:- Dio, mie' preghi exaldi, / Se t'è in piacer, o figliuol de Maria, / Che questo franco e possente Pagano / torni alla fede tua, Padre sovrano. -»; et I, 5, 1-6: «Feraù fra se stesso dice e pensa: / - [...] O vero Macometto, tu dispensa / Che questo Cristian sia al tuo comando".

54. Combattimento, II, 7, 3-5: «-O gentil conte, doname batesmo, / [...] / Vegio che non val nulla el Paganesmo»; Inamoramento, I, XIX, 13, 1-6: «- Bategiami, Barone, ala fontana / Prima che io perda in tuto la favella; / E se mia vita è stata iniqua e strana, / Non sia la morte almen de Dio rebela: / Lui che véne a salvar la gente humana, / L'anima mia ricoglia tapinella!».

55. Voir respectivement, Teseida, V, 69, 1-2; Inamoramento, I, XIX, 15, 1-2.

56. Teseida, ibid., v. 3; Inamoramento, ibid., 16, 3. 
E de pianger con seco non si saccia,

Chiedendoli perdon con voce umana.

[...]

Pregando Dio per lui con le man gionte. (Inamoramento, I, XIX, 16, 5-8)

Arcita, se croyant coupable de la mort de Palemone, exprime lui aussi sa douleur dans un poignant monologue, mais le recours à la mythologie ainsi qu'à des formules rhétoriques stéréotypées (exclamation, interrogation rhétorique) ôtent à son monologue la fraîcheur et la spontanéité qui rendent si attachant celui d'Orlando, ainsi que nous pouvons le constater en relisant la plainte d'Arcita:

[...] - Lassa omai la vita mia!

Morto è il mio compagno valoroso;

Ma di ciò testimon Febo mi sia,

Che io non fui di ciò volonteroso,

Né mai battaglia con lui disiai.

$\mathrm{O}$ me dolente, perché mai amai ${ }^{57}$ ?

Dans la Spagna F l'attention est portée à l'accomplissement des gestes du baptême, alors que dans l'Inamoramento, sur l'exemple de Teseida, la focalisation se fait sur l'émotion et le regret du héros pour avoir donné la mort. Regret d'autant plus remarquable que la victime est un ennemi, quoique courtois, loyal et courageux. L'épisode du duel entre Arcita et Palemone a vraisemblablement inspiré Boiardo pour conférer à ses personnages une épaisseur psychologique et humaine dont il ne pouvait trouver d'exemple chez les héros, encore très rudimentaires de la tradition carolingienne. Boiardo n'accueille toutefois pas la surenchère rhétorique et érudite déployée par Boccace dans Teseida.

Si nous prenons maintenant en considération un autre élément caractéristique de l'Inamoramento, les exordes, nous constatons que dans ce cas aussi, Teseida, modèle littéraire du poème d'armes et d'amour en strophes de huit vers, a été pour Boiardo un exemple fécond. Le livre VI de Teseida, qui marque le début de la partie proprement épique du poème, est introduit par un exorde contenant une réflexion de caractère général en rapport étroit avec les événements dramatiques qui vont suivre. Il s'agit du premier et unique exorde de ce genre que Boccace insère juste au milieu de

57. Teseida, V, 70, 3-8. En fait la plainte d'Arcita sur le corps inanimé de Palemone semble plutôt avoir servi de modèle pour la plainte d'Orlando sur les armes de son cousin Ranaldo qu'il croit mort: «Io te chiedo mercè, damme perdono, / Se io te offesi mai, dolce germano, / Ch' io fui pur sempre tuo, come ora sono, / Benché falso suspetto ed amor vano / E l'arme zelosia ce pose in mano" (Inamoramento, II, VII, 52, 1-5). 
son œuvre pour marquer un tournant capital dans l'inspiration et dans le ton. De même, dans l'Inamoramento il faut attendre le chant XVI du

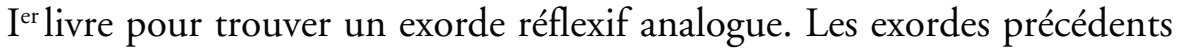
contenaient essentiellement des adresses au public ou de brefs rappels des événements. Boiardo lui aussi, arrivé au milieu d'un livre qui compte en tout XXIX chants, sent donc la nécessité de marquer un tournant. En effet, à partir du chant XVI, l'attention se focalise sur Agricane, héros dont on a déjà beaucoup parlé. Avec ce personnage, la mort fait son entrée tragique dans l'univers romanesque de l'Inamoramento et Boiardo, tout comme Boccace dans Teseida, souligne l'important changement de ton et de veine que son poème va connaître en faisant de son exorde un moment de réflexion. Nous savons qu'il s'agit par ailleurs d'un procédé que l'Arioste va généraliser. Les coïncidences vont même plus loin, car non seulement Boiardo a trouvé dans Teseida l'idée d'un exorde réflexif, mais il en a également emprunté le sujet. Son premier exorde-réflexion, tout comme celui du livre VI de Teseida, traite en effet de la fortune et de son instabilité:

L'alta ministra del mondo Fortuna,

Con volubile moto permutando

Di questo in quel più volte ciascheduna

Cosa togliendo e tal volta donando,

Or mostrandosi chiara e ora bruna

Secondo le pareva e come e quando,

Avea co'suoi effetti a'due Tebani

Mostrato ciò che può ne' ben mondani. (Teseida, VI, 1)

Tutte le cose sotto dela luna,

L'alta richeza, e regni dela terra

Son sotoposti a voglia di Fortuna:

Lei la porta apre de' improviso, e sèra,

E quando più par bianca divien bruna;

Ma più se mostra a caso dela guerra

Instabile, voltante e roïnosa,

E più falace che alcuna altra cosa. (Inamoramento, I, XVI, 1)

À cette analogie thématique de fond s'ajoutent les similitudes plus ponctuelles des images véhiculant l'idée du mouvement et du changement perpétuel de la fortune. Plus précisément, l'image de la fortune virant sans transition du blanc au noir, présente autant chez Boccace que chez Boiardo, est récurrente dans la culture française du Moyen Âge. Ici, la fortune était présentée tantôt avec une veste, voire les membres, des deux couleurs, tantôt sous la forme de deux personnifications indépendantes, 
l'une blanche et l'autre noire ${ }^{58}$. La portée de la réflexion de Boiardo est plus générale que celle de Boccace et va au-delà de la situation spécifique des personnages concernés pour devenir une méditation sur l'action de la fortune sur la vie humaine, problématique qui est au cœur du roman du comte de Scandiano. Malgré cela, le rôle exercé par Teseida sur cet exorde de l'Inamoramento est incontestable.

Quelques exordes de Teseida décrivent la régénération de la nature sous l'effet bénéfique du printemps ou bien de changements climatiques (II, 1, IV, 1). Boiardo s'en inspire dans certains de ses débuts de chant, mais il confere à ces descriptions convenues une tout autre portée idéologique. Ainsi dans l'exorde du chant I du livre II de l'Inamoramento retrouve-t-on des emprunts descriptifs au poemetto de Boccace:

Nel dolce tempo che il ciel fa belle
Le valli e' monti d'erbette e di fiori,
E le piante riveste di novelle
Frondi, sopra le quali i loro amori
Cantan gli uccelli, e le gaie donzelle
Di Citerea più senton gli ardori. (Teseida, II, 3, 1-6)
Nel gratioso tempo onde natura
Fa più lucente la stella d'amore,
Quando la terra copre di verdura
E li arboseli adorna di bel fiore,
Gioveni e dame et ogni creatura
Fano alegreza con zoglioso core. (Inamoramento, II, 1, 1-6)

Dans l'Inamoramento, la représentation du printemps devient la métaphore de la renaissance des valeurs chevaleresques et courtoises à la cour de Ferrare et donc un moyen d'en célébrer indirectement les seigneurs. Cet exorde, en effet, ouvre le livre II axé sur le personnage de Ruggiero, descendant d'Alexandre le Grand et lui-même ancêtre de la maison d'Este. Nous savons qu'en introduisant Ruggiero, Boiardo, comme déjà Tito Vespasiano Strozzi dans la Borsias, œuvre à la gloire de Borso d'Este, s'engageait à effacer une généalogie peu flatteuse circulant à l'époque sur les Este et qui les faisait descendre de Ganelon de Mayence, le traître de Roncevaux. Sur ce motif encomiastique s'en greffe un autre d'ordre historique, car le renouvellement printanier dont il est question dans l'exorde célébrerait, d'après des recherches nouvelles, l'avènement ou le tout début $\mathrm{du}$ règne d'Hercule, présenté comme une époque de renaissance et d'espoir. Aux yeux du comte de Scandiano, la cour d'Hercule I ${ }^{\text {er }}$ incarne

58. Voir Inamoramento, vol. I, p. 464, note 1. 
de fait une sorte de Camelot moderne $\mathrm{e}^{59}$ où les idéaux traditionnels de la chevalerie et de la courtoisie ont trouvé non seulement un accueil adéquat, mais aussi un autre essor grâce à l'action culturelle de seigneurs éclairés qui ont su concilier les nouvelles valeurs de la culture humaniste avec celles de l'ancienne chevalerie.

Nous comparerons, pour conclure, une strophe de l'exorde général de Teseida avec le début de l'exorde du chant XII du livre II de l'Inamoramento:

Siate presenti, o Marte rubicondo,

Nelle tua armi rigido e feroce,

E tu, madre d'Amor, col tuo giocondo

E lieto aspetto, e 'l tuo figliuol veloce

Co' dardi suoi possenti in ogni mondo;

E sostenete e la mano e la voce

Di me che'ntendo i vostri effetti dire

Con poco bene e pien d'assai martire. (Teseida, I, 3)

Stela d'Amor che 'l terzo ciel governi,

$\mathrm{E}$ tu, quinto splendor si rubicondo

Che girando in dui anni e cerchi eterni

D’ogni pigricia fai digiuno il mondo,

Venga da' corpi vostri alti e superni

Gratia e vertute al mio cantar iocondo

Sì che l'influxo vostro hor mi vaglia

Poi che de Amor canto e di bataglia. (Inamoramento, II, XII, 1)

Dans ce cas aussi, comme dans les précédents, on constate une analogie thématique de fond - l'invocation à Mars et à Vénus, les divinités protectrices d'une entreprise poétique qui chante l'amour et les armes - et d'autres plus ponctuelles, notamment la reprise des mêmes adjectifs (rubicondo, giocondo) en fin de vers. Mais ici encore, Boiardo enrichit la portée symbolique de son exorde par rapport à celui de son modèle. La «Stela d'Amor» dont il est question au début de son chant - observons en passant que Boccace débute par une invocation à Mars - n'est pas simplement la déesse Vénus; elle ferait allusion à l'amour d'origine divine dont parle Dante au début du Paradis. De même, Mars n'est pas présenté en tant que dieu de la guerre, de l'affrontement épique des armées, mais en tant que protecteur de l'activité des armes, propre au chevalier et à l'homme de cour, et donc la seule digne de louange selon l'optique courtoise de Boiardo ${ }^{60}$.

Tous les exemples cités confirment l'éclectisme de l'inspiration de

59. L'expression est de Riccardo Bruscagli, et figure dans l'introduction à son édition de M.M. BoIARDO, Orlando innamorato, Turin, Einaudi, 1995, 2 vol., vol. I, p. XX.

60. Voir Inamoramento, vol. II, p. 1104, note 1. 
Boiardo qui prend son bonheur là où il le trouve, que ce soit chez Boccace ou dans la tradition carolingienne. Teseida a toutefois été pour le comte de Scandiano un point de repère fort pour la partie proprement épique de son poème. Il y a trouvé de quoi enrichir les dimensions humaine et psychologique de ses personnages ainsi que des exemples concrets pour transformer l'exorde de simple introduction du récit en un moment de réflexion capable de conférer une valeur exemplaire et philosophique à l'histoire présentée.

\section{Éléments stylistiques et linguistiques}

Considérons, avant de conclure, l'impact que Boccace a eu sur certains aspects du style et du lexique de Boiardo. Pour commencer par l'examen de quelques similitudes, prenons une comparaison épique courante, dont l'archétype se trouve dans l'Énéide (X, 707-718), mettant en rapport le comportement d'un combattant avec celui d'un sanglier traqué par les chiens:

Qual per lo bosco il cinghiar ruvinoso,

Poi c’ha di dietro a sé sentiti i cani,

Con le sete levate e isquamoso,

Or qua or là per viottoli strani

Rugghiando va fuggendo furioso,

Rami rompendo e schiantando silvani,

Cotale entrò mirabilmente armato

Palemon quivi da ciascun mirato. (Teseida, VII, 119) ${ }^{61}$

E come un cingial tra can mastini,

Che intorno se ragira furioso

E nel fronte superbo adriza e crini,

E fa la schiuma al dente sanguinoso;

Sembrano un foco li ochi picolini,

Alcia le sete e sanza alcun riposo

La fiera testa fulminando mena:

Chi più se gli avicina ha magior pena;

Non altramenti, quella dama altiera

De driti e de riversi oltra misura

Facea bataglia sì crudel e fiera. (Inamoramento, I, XIX, 45, 46, 1-3)

Malgré la présence de quelques éléments communs - les chiens, les poils

61. On trouve une similitude analogue in Teseida, I, 38: «Né altramenti il cinghiar c'ha sentiti / Nel bosco i can fremire e'cacciatori, / I denti batte e rugghia e gli spediti / Sentieri a sua salute cerca e, pe' romori / Ch'egli ha in qua in là in giù e 'n su uditi, / Non sa qua' vie per lui si sien migliori, / Ma ora in giù e ora in su correndo, / Fino al bisogno, incerto, va fuggendo ». 
hérissés - les deux représentations sont très différentes. Boiardo, qui recourt à cette image pour décrire la guerrière Marfisa, se concentre sur la figure menaçante de l'animal (il a l'écume à la bouche, les yeux petits et injectés de sang). Boccace, en revanche, qui s'en sert pour caractériser Palemone entrant dans l'arène des combats, s'arrête sur l'environnement de l'animal (il s'enfuit dans le bois, il brise des branches). Également peu probant nous paraît le rapprochement établi entre la similitude du sanglier de Boiardo et une autre de même sujet figurant dans un poème chevaleresque anonyme, l'Orlando $H$. Les quelques coïncidences lexicales entre le texte de Boiardo et celui de l'auteur anonyme ne sont pas assez significatives pour permettre de reconnaître une dérivation. Les correspondances les plus intéressantes se remarquent en effet à travers certaines tournures particulièrement expressives très répandues dans les poèmes en ottava rima que Boiardo récupère avec habileté et ironie ${ }^{62}$. Les mêmes observations peuvent valoir pour une autre similitude épique courante. Il s'agit de la représentation de la rivière qui dévale une pente pour rendre la violence d'un combat:

Giungersi sì le schiere di costoro

Con corsi più veloci e più perversi

Che d'alto monte, per subita piova,

Rabbioso il rivo il pian letto ritrova. (Teseida, VIII, 6, 5-8)

E comme un rivo da il monte declina

Con sassi rotti et arbori dispersi,

Cossì quei doi Baron pien di valore

Se urtarno con grandissimo romore. (Inamoramento, I, XXVIII, 13, 5-8)

L'analogie ne va pas au-delà du niveau thématique; rien ni dans le lexique, à part le mot rivo, ni plus généralement dans l'expression ne permet de dégager une dérivation précise d'un texte à l'autre. Les mêmes remarques sont valables aussi pour une similitude lyrique aussi courante que les précédentes: la comparaison entre l'amoureux qui reprend espoir et la nature qui se réveille. Il s'agit à l'origine d'une image provenant de Dante (Enfer, II, 127-132) que Boccace reprend assez fidèlement dans Filostrato et Teseida et que nous retrouvons transformée chez Boiardo, comme le montrent les exemples:

Quali i fioretti, dal notturno gelo

62. Pour le rapprochement entre les similitudes du sanglier chez Boiardo et dans l'Orlando $H$, voir R. Donnarumma, "Presenze... », cit., p. 540-542. Il est par ailleurs intéressant d'observer l'analogie entre le vers "E rugge e batte forte dente con dente», se référant au sanglier dans l'Orlando $H$ et cet autre vers de Boiardo: "Ma rodendo si va l'ongie col dente", où il est question de l'impatience jalouse d'Orlando (I, XXV, 61, 4). 
Chinati e chiusi, poi che 'l sol gl'imbianca,

Tutti s'apron diritti in loro stelo,

Cotal si fe di sua virtute stanca

Troiolo allora [...]. (Filostrato, II, 80, 1-5) ${ }^{63}$

Comme doppo la piogia le viole

Se abbatino, e la rosa, e il bianco fiore,

Poi quando al ciel sereno appare il sole,

Apren le foglie e torna il bel colore,

Cossì Prasildo ala lieta novella

Dentro si alegra e nel viso se abella. (Inamoramento, I, XII, 85, 3-8)

Il est donc difficile d'indiquer l'origine de ces images très répandues dans la tradition poétique italienne. Un autre exemple allant dans ce sens nous vient de l'emploi métaphorique du terme conforto, désignant l'être aimé autant chez Boccace que chez Boiardo dans des expressions comme " Colui che è il mio ben e il mio conforto", "Che sola ène il mio ben e il mio conforto ». Cette métaphore, employée par Boccace, n'est pas moins récurrente dans les vers de Pétrarque, aussi est-il difficile d'en dégager la provenance chez l'auteur des Amorum Libri ${ }^{64}$.

La confrontation est apparemment plus productive si nous nous tournons vers des emprunts ponctuels de type formulaire ou lexical. Nous pouvons observer d'intéressantes coïncidences dans le domaine de certaines expressions proverbiales, comme celles qui reviennent sous la plume des deux auteurs pour désigner la toute puissance de l'amour: «Perché descriver non si può l'amore / A chi nol senti e a cui non l'ha nel core" (Inamoramento, I, XII, 10, 7-8), "Né della mia pietà qui far menzione / Più non intendo, né del loro amore, / Non conosciuto da chi non l'ha in core" (Teseida, VII, 3, 6-8). Toute-puissance qui permet à la passion amoureuse de survivre même à la mort: "Se quel morir de amor l'alma non priva», «Se, per morire, amor non se n'oblia ${ }^{65}$ ». L'utilisation d'expressions appartenant au champ sémantique du temps, du type «e ogni giorno lor pareva cento", "ciascun giorno cento parea lor" de Boccace, qui donnent chez Boiardo "uno atimo cento anni li rileva", "ché ciascuna ora li parea ben cento", "e parli un giorno cento" suscite des

63. Voir aussi Teseida, IX, 28.

64. Voir, respectivement, Teseida, XI, 5, 8 et Inamoramento, I, XXI, 44, 2; sur les emprunts de Boiardo à Pétrarque, voir Pier Vincenzo Mengaldo, La lingua del Boiardo lirico, Florence, Olschki, 1963, p. 324.

65. Inamoramento, I, XII, 54, 3 et Filostrato, IV, 54, 8. Appartiennent à une catégorie proche les vers suivants: "Che altro ristor non spera che la morte» (Inamoramento, I, XII, 16, v. 8); «Sol per ristoro chiedendo la morte" (Filostrato, VII, 11, 8), ou encore "[...] se 'l cuor non erra" (Teseida, I, 46, 7); «se 'l mio cor non erra» (Inamoramento, I, I, 16, 7). 
remarques analogues ${ }^{66}$. Il est vrai que ces expressions ne sont pas très recherchées, et qu'il s'agit soit de formes proverbiales, donc récurrentes dans la langue parlée, soit plus généralement de formes peu littéraires. Il est toutefois intéressant d'observer qu'elles occupent souvent chez les deux auteurs la même position à l'intérieur de la strophe. C'est aussi le cas d'une formule qui, autant chez Boccace que chez Boiardo, est placée à la fin d'une strophe pour marquer la conclusion du discours d'un personnage qui attend la réponse de son interlocuteur dans la strophe suivante: «Deh, non mi far di questa grazia niego", "Che ala dimanda mia non faci nego ${ }^{67}$ ». Cette identité de position dans l'ottava est un élément qui conforte l'idée d'une dérivation de Boccace à Boiardo.

L'influence est plus facile à déterminer lorsqu'il s'agit d'une image plus rare, comme l'expression originale utilisée par Boccace pour décrire l'entrée de la demeure du dieu Mars: "tutta di ferro era la stretta entrata» (Teseida, VII, 32, 6), et qui est reprise telle quelle ou avec de légères variantes par Boiardo ${ }^{68}$. Pour en rester au domaine des termes rares, on peut encore signaler l'utilisation par les deux auteurs du verbe "sdruscire» pour désigner l'effet destructeur de la tempête sur un navire ${ }^{69}$. Dans un tout autre registre, on signalera le réemploi avec variante de la formule obscène de Boccace "scuotere il pelliccione ${ }^{70}$ ", dans une fameuse plainte où Orlando déplore sa propre maladresse érotique:

E se io volesse alciar una pelicia

Di donna, io non sarìa morto né vivo:

Se lei non me insignasse o desse ardire,

Cominciar non saprebbe io, né finire. (Inamoramento, I, XXV, 57, 5-8)

Il nous reste à prendre en examen, pour finir, quelques emprunts lexi-

66. Teseida, III, 46, 5; VI, 10, 2-3 et Inamoramento, I, II, 27, 5; I, IV, 13, 3; I, XII, 42, 4. Appartiennent à cette catégorie temporelle aussi les vers suivants: "Il giorno inverso della notte andava", "Il giorno [...] sen giva in ver la sera" (Teseida, XI, 57, 1 et XII, 75, 1-2); «E ver la nòte se n'andava il giorno", "Il giorno si calava in ver la sera" (Inamoramento, I, XXVIII, 37, 6 et I, I, $59,1)$.

67. Voir, respectivement, Filostrato, I, 55, 8; Inamoramento, I, IV, 5, 8; voir aussi : «[...] io te ne priego / che or di questo non mi facci niego" (Filostrato, II, 112, v. 8); «E al mio domandar non metter niego ", "Ch'alli miei prieghi tu non facci niego" (Teseida, IV, 45, 8 et VII, 25, 8). Sur les clauses de fin de strophe chez Boccace et Boiardo, voir R. Donnarumma, "Presenze...", cit., p. 535.

68. " $\mathrm{E}$ un bel giardin et ha di fero il muro", "Tuta de calamita era la intrata» (Inamoramento, I, XII, 26, 6; 41, 1) ; signalons aussi, dans le domaine des analogies descriptives: «Era la terra forte e ben murata» (Teseida, I, 78, 1), repris par Boiardo avec une légère variante syntaxique: «Forte è la terra, intorno ben murata" (Inamoramento, I, IV, 59, 7).

69. "Sentirono la nave sdruscire», «sopra la isdruscita nave si gittarono i padroni», «E la nave [...] quantunque sdruscita fosse» (Decameron, II, 7, 11-13); "Altro non se ode che nave strusire / Et alti gridi e pianti da morire" (Inamoramento, II, VI, 34, 7-8).

70. Voir Decameron, IV, 10, 46, VIII, 7, 103, X, 10, 69. 
caux que Boiardo a faits à Boccace ${ }^{71}$. Les emprunts les plus fréquents et significatifs concernent certains adjectifs. Le qualificatif membruto utilisé maintes fois par Boiardo et récurrent dans le poème chevaleresque pour souligner la prestance et les belles proportions d'un personnage masculin, a son archétype, pour ainsi dire, chez Boccace qui l'introduit dans Teseida pour décrire Palemone: "Era Palemon grande e ben membruto,/ Brunetto alquanto e nello aspetto lieto, / [...] Di pel rossetto e assai grazioso $^{72}$ ». Parmi les adjectifs expressifs, introduits par Boccace pour définir un état psychologique particulier, se signalent «fellone e niquitoso» (Filostrato, V, 15, 3) et le terme musorno. Les deux premiers qualifient un état d'âme de forte irritation et sont repris par Boiardo dans une acception analogue $^{73}$. En revanche musorno, qui signifie triste, maussade chez Boccace (Teseida, XI, 17, 1: «in quella stando sì come musorno»), est une injure chez Boiardo, utilisée dans cette acception dans la Spagna F et plus généralement dans le roman chevaleresque ${ }^{74}$. L'adjectif guazzoso, employé par Boccace dans la description du climat pluvieux et humide de l'hiver et réutilisé dans l'Inamoramento dans une acception identique, "Come de verno nel tempo guazoso ${ }^{75}$ ", appartient à la même catégorie de termes intéressants. L'épithète de tradition florentine ignudanata, signifiant «nue comme à la naissance», se trouve dans une nouvelle du Decameron où un prêtre rusé essaye de profiter de la femme d'un mari plutôt naïf. Boiardo le réemploie dans un exorde de son poème pour indiquer la nudité complète d'un personnage monstrueux - «Era quel' Orione ignudonato» - le transférant ainsi d'un contexte populaire et comique à un contexte merveilleux $^{76}$. L'adjectif lavorata, associé par Boccace au mot acqua, dans le sens d'eau mélangée à d'autres substances (Dec., VII, 3, 10), est repris par

71. Pour le lexique de l'Inamoramento, on se reportera à l'ouvrage de Domizia Trolli, Il lessico dell'Inamoramento de Orlando di Matteo Maria Boiardo, Milan, Unicopli, 2003.

72. Teseida, III, 49 à comparer avec les lieux suivants de l'Inamoramento: «Era questo re grande e ben membruto" (I, IX, 48, 3), "Ch'era un cavalier grande e ben membruto" (I, XX, 5, 3), " Ordauro era di forza più virile / E grande di persona e ben membruto " (I, XXI, 52, 5-6); voir également, toujours chez Boiardo et à comparer avec la description de Palemone: «Brunetta alquanto e grande di persona" (dit de Marfisa, I, XXVII, 59, 7), "Di pel rossetto et aquilino in faza» (II, $\mathrm{XXV}, 54,5)$.

73. Inamoramento, I, II, 22, 1-4: «Ma comme [Orlando] intese che egli era fugito / L'Argalia al bosco e seco la dongella, / E che Ranaldo lo aveva seguito, / Partisse in vista nequitosa e fella».

74. Inamoramento, I, II, 13, 5: «Poi che hagio il libro di quel can musorno»; voir aussi Spagna F, VIII, 44.

75. Inamoramento, I, X, 53, 1, à comparer avec "Quanto può fare il tempo più guazzoso", « in varii luoghi ne' guazzosi verni" (Teseida, respectivement, IV, 1, 1 et VII, 30, 5).

76. Inamoramento, I, V, 1, 6; Decameron, IX, 10, 17: «Appresso donno Gianni fece spogliare ignudanata comar Gemmata». 
Boiardo pour qualifier le breuvage que la fée Febosilla administre au cheval Batoldo blessé: "Con succi d'herbe et aqua lavorata" (Inamoramento, II, XXVI, 19, 4).

D’après les sondages effectués qui, certes, ne prétendent pas à l'exhaustivité, il résulte que Boiardo a tiré de Boccace surtout des formules proverbiales, des expressions idiomatiques et populaires, des termes au caractère phonique particulièrement marqué (guazzoso, musorno, ignudonato). Certains qualificatifs utilisés par Boccace, transférés dans la tradition chevaleresque en ottava rima, ont perdu partiellement ou totalement leur signification originaire pour en assumer une autre plus générique, devenant ainsi des outils très pratiques pour les poètes engagés comme Boiardo dans des ouvrages de très longue haleine ${ }^{77}$. D'un point de vue stylistique général, en revanche, l'influence de Boccace sur Boiardo ne nous paraît pas avoir été particulièrement significative en raison, certainement, de la différence de ton et de genre existant entre l'Inamoramento et les trois œuvres de Boccace considérées.

Dans l'ensemble, nous avons constaté que l'influence de Filostrato, de Teseida et du Decameron se manifeste surtout dans le $\mathrm{I}^{\mathrm{er}}$ livre de l'Inamoramento, beaucoup moins dans les deux autres. Cela ne surprend pas étant donné que le sujet amoureux, dominant dans ces trois œuvres de Boccace, l'est aussi dans le livre I de Boiardo axé sur le renversement parodique du rôle d'Orlando. En ce qui concerne plus spécifiquement le Decameron, son influence est particulièrement évidente, et cela n'est pas pour nous surprendre, dans certaines des sept nouvelles de l'Inamoramento, notamment celles d'Iroldo, Tisbina et Prasildo, ainsi que celle de Leodilla et Ordauro (livre I, chants XXI et XXII). Ailleurs, la présence du Decameron est beaucoup plus irrégulière et, ce qui est significatif, concerne essentiellement le niveau expressif et lexical.

En considérant les poemetti, on constate qu'ils sont eux aussi très présents dans la nouvelle courtoise d'Iroldo, Tisbina et Prasildo, qui s'impose comme le lieu de l'Inamoramento où la trace de Boccace est la plus forte. Quant à Teseida, en dehors de la nouvelle d'Iroldo, Tisbina et Prasildo, son influence s'exerce surtout sur certains exordes de l'Inamoramento et dans les chants XVIII et XIX du premier livre consacrés au duel entre

77. Voir D. Trolli, Il lessico dell'Inamoramento, cit., p. 23-24. 
Orlando et le roi païen Agricane. Teseida, poème à la double inspiration amoureuse et guerrière, est en effet une source choisie pour les chants où il est question de combats.

Même si nous savons et avons montré que Boiardo pratique l'éclectisme des sources comme peu d'auteurs l'ont fait, l'influence de Boccace sur l'Inamoramento se dégage avec netteté. Il est certain que le lyrisme des poemetti, repris par Boiardo, assez souvent, mais pas uniquement, dans une intention parodique, a considérablement enrichi la psychologie et l'expression des héros carolingiens du roman du comte de Scandiano. Mais ce dernier possédait déjà dans le domaine de l'épanchement amoureux subjectif une solide expérience dont témoignent les Amorum Libri Tres. Nous voudrions surtout souligner le rôle déterminant de l'expérience de Boccace au niveau de la dimension réaliste et populaire, autrement dit en tant qu'inventeur de la «letteratura mezzana». Nous savons en effet que revient à Boiardo l'idée d'agrémenter le récit chevaleresque par l'introduction de nouvelles. Comment ne pas penser que cette idée lui soit venue suite à la fréquentation assidue de l'auteur du Decameron? Laissant de côté les emprunts directs au chef-d'œuvre de Boccace, nous avons pu voir combien le comte de Scandiano est friand de situations et d'expressions provenant de ce genre littéraire. Il aime bien emprunter à Boccace les situations, les formules et les termes qui frappent l'esprit et les sens de son public et qui, introduits dans le contexte de son roman, se modifient, se transforment, assument des significations inédites. Bref, chez l'inventeur de la «letteratura mezzana», Boiardo a d'abord trouvé ce goût pour le mélange entre le haut et le bas, entre le noble et le populaire qui est également au cœur de son entreprise. Paradoxalement, la profondeur de l'influence exercée par Boccace sur Boiardo, se mesure davantage sur des éléments fragmentaires et de détail comme le sont certains aspects de l'expression ou du lexique que sur de longs morceaux. Nous avons pu ainsi constater, l'apport relativement modeste de Boccace au niveau de certaines figures de style classiques. Mais n'est-ce pas ceci la preuve d'une connaissance approfondie et menue d'une œuvre et d'un auteur avec lesquels le comte de Scandiano sent beaucoup d'affinités? 